\title{
Intravenous Immunoglobulins Lower Inflammatory Gene Expression in Skin Biopsies of Chronic Inflammatory Demyelinating Polyradiculoneuropathy Patients
}

\author{
Pinelopi Tsouni Petrica-Adrian Panaite Stefania Puttini Thierry Kuntzer \\ Andreas J. Steck
}

Department of Clinical Neurosciences, Nerve-Muscle Unit, Neurology Service, Lausanne University Hospital (CHUV), Lausanne, Switzerland

Dear Sir,

Chronic inflammatory demyelinating polyradiculoneuropathy (CIDP) is the most common form of chronic autoimmune neuropathy [1]. The underlying immune-mediated mechanisms have not yet been fully elucidated [2], but gene expression analyses identified inflammatory molecular markers that are upregulated in skin nerve biopsies $[3,4]$. Intravenous immunoglobulins (IVIG) are a first-line therapy for CIDP [5]. In order to gain insight in the anti-inflammatory effects of IVIG, 2 subgroups of patients treated with either IVIG or other immunosuppressive agents were selected from a previous gene expression profiling study [4]. The selected subgroups were further studied using a transcriptional microarray analysis in skin punch biopsies.

Four patients ('IVIG' group) were treated with Privigen ${ }^{\circledR}(2 \mathrm{~g} / \mathrm{kg}$ administered over 5 days) 2-6 months before skin biopsy was performed. Three patients ('NoIVIG' group) received other treatments: prednisone (30 mg/day), azathioprine $(2 \mathrm{mg} / \mathrm{kg} /$ day) or tacrolimus ( $2 \mathrm{mg} /$ day), respectively. All 7 patients had stable active disease according to CIDP Disease Activity Status (CDAS) [4, 6]. Twelve healthy volunteers were used as controls ('Ctrl' group). Skin punch biopsies were performed $10 \mathrm{~cm}$ above the external malleoli and snap frozen in liquid nitrogen. RNA extraction, gene expression profiling, and MetaCore ${ }^{\circledR}$ data analysis were performed according to the methods described previously [4] with the following filters: fold expression change $>1.15, \mathrm{p}<0.05, \mathrm{Y}$ chromosome linked genes and duplicate removal. Data analysis was made according to a 3 set comparison (C), where C1 stands for 'IVIG vs. Ctrl', C2 for 'IVIG vs. NoIVIG', and C3 for 'NoIVIG vs. Ctrl'. This study was approved by the local Ethics Committee (protocol 235/10).

We first analyzed the total number of differentially regulated genes between the 3 groups. Results showed 223 differentially regulated genes between patients treated with IVIG and the control group $(\mathrm{C} 1$; fig. 1a). In contrast, numbers of differentially regulated genes were much higher when comparing NoIVIG patients to IVIG and the control group respectively: 1117 genes were differentially regulated between patients treated with IVIG vs. NoIVIG (C2) and 1022 genes were differentially regulated between NoIVIG vs. controls (C3) (online suppl. tables C1, C2 and C3, see www. karger.com/doi/10.1159/000447127).

A downregulation of genes involved in inflammation, tissue remodeling and wound repair and cell-cycle regulation such as ULK3 (control of autophagy), TRAP1 (stress regulation), ANAPC11 (cellcycle regulation), CEBPA (transcription factor), $P D R G 1$ (inflammation), $P F K L$ (AKT signaling), and NCS1 (calcium sensor) was found in IVIG-treated patients (fig. 1b). All these genes were downregulated in IVIG-treated patients (comparisons $\mathrm{C} 1$ and $\mathrm{C} 2$ ) but not in the non-IVIGtreated subgroup (comparison C3). In addition, in comparison to C2, IVIG treatment was found to downregulate $A K T$ (fold change $=-1.38, \mathrm{p}=0.01$ ) as well as its downstream partners CyclinD1 and CDK4, which are the key regulators of the cell cycle.

T.K. and A.J.S. are equal senior authors.

\section{KARGER}

E-Mail karger@karger.com

www.karger.com/ene (c) 2016 S. Karger AG, Base

0014-3022/16/0756-0290\$39.50/0
Thierry Kuntzer, MD or Andreas J Steck, MD Department of Clinical Neurosciences Nerve-Muscle Unit, Lausanne University Hospital (CHUV) Rue du Bugnon 46, CH-1011 Lausanne (Switzerland)

E-Mail thierry.kuntzer@chuv.ch or andreas.steck@unibas.ch 
Fig. 1. a The histograms (white, gray and black) represent the total numbers of differentially regulated genes (vertical axis) in the 3 groups compared (horizontal axis) according to the MetaCore ${ }^{\circledR}$ analysis. b The histograms (white, gray and black) represent the fold changes (vertical axis) of genes involved in inflammation, tissue remodeling and wound repair and cell-cycle regulation (horizontal axis). These genes were down-regulated in $\mathrm{C} 1$ and $\mathrm{C} 2$ comparison (negative fold changes), but in C3 (positive values).

Taken together, our results suggest that IVIG-treated patients have a gene profile (fig. 1a) that is more similar to that of healthy subjects than that of patients treated with other immunosuppressants. In addition, our data show the downregulation of genes involved in inflammation and proliferation (fig. 1b), genes previously reported to be upregulated in active CIDP $[3,4]$. Of particular interest, is the downregulation of $A K T$, a key cell-cycle regulator [7] and its downstream partners CyclinD1 and CDK4 by IVIG therapy. This suggests a lower activity of cell proliferation in patients treated with IVIG compared to other immunosuppressants. Based on this find-

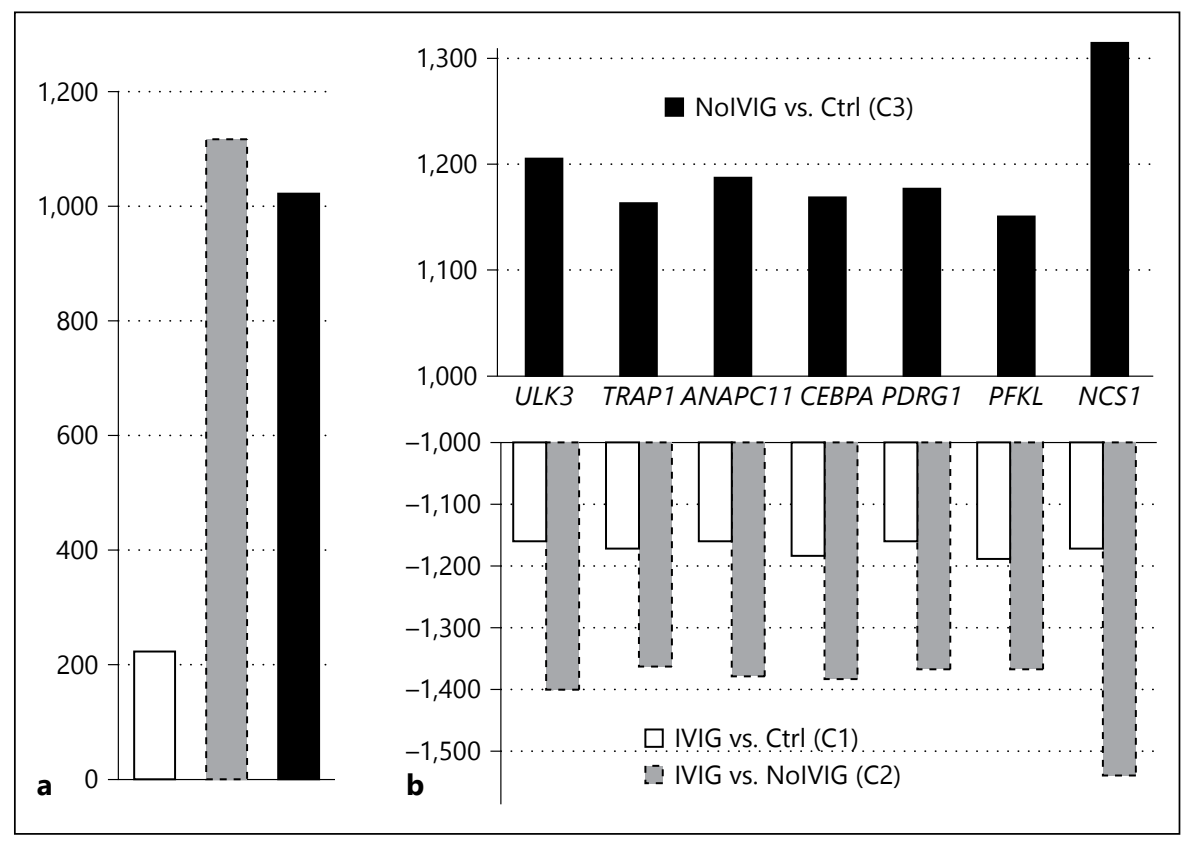

ing, and on reports that IVIG inhibits Tcell proliferation [8], we speculate that IVIG therapy may inhibit T-cell proliferation in inflamed nerve fibers in CIDP patients [2]. Lower maintenance doses of non-IVIG immunosuppressants used to treat our CIDP patients may explain why these agents were not shown to lower inflammatory expression in our study. The fact that all patients had stable active disease according to their CDAS score independent of treatment argues against a disease-related confounding effect. Whether the timing of skin biopsy influences these observations remains to be demonstrated in a larger cohort of patients.

\section{Acknowledgments}

This work was supported by a grant from NeRAB (www.nerab.org) and by an unrestricted contribution from CSL-Behring.

\section{Statement of Ethics}

The study has been approved by the Ethics Committee.

\section{Disclosure Statement}

There is no conflict of interest in relation to this work.

\section{References}

1 Mathey EK, Park SB, Hughes RA, et al: Chronic inflammatory demyelinating polyradiculoneuropathy: from pathology to phenotype. J Neurol Neurosurg Psychiatry 2015;86: 973-985.

2 Dalakas MC; Medscape: Advances in the diagnosis, pathogenesis and treatment of CIDP. Nat Rev Neurol 2011;7:507-517.

3 Lee G, Xiang Z, Brannagan TH 3rd, Chin RL, Latov N: Differential gene expression in chronic inflammatory demyelinating polyneuropathy (CIDP) skin biopsies. J Neurol Sci 2010;290:115-122.

4 Puttini S, Panaite PA, Mermod N, Renaud S, Steck AJ, Kuntzer T: Gene expression changes in chronic inflammatory demyelinating polyneuropathy skin biopsies. J Neuroimmunol 2014;270:61-66.

5 Nobile-Orazio E, Gallia F: Update on the treatment of chronic inflammatory demyelinating polyradiculoneuropathy. Curr Opin Neurol 2015;28:480-485.
6 Panaite PA, Renaud S, Kraftsik R, Steck AJ, Kuntzer T: Impairment and disability in 20 CIDP patients according to disease activity status. J Peripher Nerv Syst 2013;18:241-246.

7 Liang J, Slingerland JM: Multiple roles of the $\mathrm{PI} 3 \mathrm{~K} / \mathrm{PKB}$ (Akt) pathway in cell cycle progression. Cell Cycle 2003;2:339-345.

8 MacMillan HF, Lee T, Issekutz AC: Intravenous immunoglobulin G-mediated inhibition of T-cell proliferation reflects an endogenous mechanism by which IgG modulates T-cell activation. Clin Immunol 2009;132: 222-233. 